\title{
EGU21-2248
}

https://doi.org/10.5194/egusphere-egu21-2248

EGU General Assembly 2021

(c) Author(s) 2022. This work is distributed under

the Creative Commons Attribution 4.0 License.

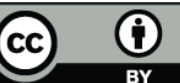

\section{Identifying mesoscale high-wind features within extratropical cyclones}

Lea Eisenstein, Peter Knippertz, and Joaquim G. Pinto

Institute of Meteorology and Climate Research, Karlsruhe Institute of Technology, Karlsruhe, Germany

(lea.eisenstein@kit.edu)

Extratropical cyclones cause strong winds and heavy precipitation events and are therefore one of the most dangerous natural hazards in Europe. The strongest winds within these cyclones are mostly connected to four mesoscale dynamical features: the warm (conveyor belt) jet (WJ), the cold (conveyor belt) jet (CJ), cold-frontal convective features (CFC) and the sting jet (SJ). While all four have high wind gust speeds in common, the timing, location and some further characteristics typically differ and hence likely also the forecast errors occurring in association with them.

Here we present an objective identification approach for the four features named above based on their most important characteristics in wind, rainfall, pressure and temperature evolution. The main motivations for this are to generate a climatology for Central Europe, to analyse forecast error specific to individual features, and to ultimately improve forecasts of high wind events through feature-dependent statistical post-processing. To achieve, we ideally want to be able to identify the features in surface observations and in forecasts in a consistent way.

Based on a dataset of hourly observations over Europe and nine windstorm cases during the winter seasons 2017/18, 2018/19 and 2019/20, it became apparent that mean sea-level pressure tendency, potential temperature tendency, change in wind direction and precipitation (all onehourly) are most important for the distinction between the WJ and CFC. Further adding the time (relative to storm evolution) and location (relative to the storm centre) of occurrence helps to identify the CJ. Ultimately, the identification of each feature is based on a score on a scale from 0 to 10 that reflects the various criteria for a station or grid point. Additionally, exclusion criteria for each feature are defined to rule out locations that meet some criteria (and thus have a positive score) but strongly violate others. Finally, smooth contours are drawn around each feature to define their spatial extent.

While the distinction between WJ and CFC seems to work reliably, the identification of CJ remains ambiguous and needs further parameters and exclusion criteria to avoid too large areas and overlap with other features. Furthermore, SJ and CJ are very difficult to distinguish based on surface observations alone and are therefore taken together for this preliminary analysis. Once the definition of criteria is finalised, a climatology will be compiled based on observations and the German COSMO model and forecast errors analysed for said model. 
